\title{
The Role of Medications for the Management of Patients with NAFLD
}

\author{
Natalia Mazzella, MD ${ }^{a}$, Laura M. Ricciardi, $\mathrm{MD}^{\mathrm{b}}$, \\ Arianna Mazzotti, MD ${ }^{a}$, Giulio Marchesini, $\mathrm{MD}^{\mathrm{c}, *}$
}

\section{KEYWORDS}

- Nonalcoholic fatty liver disease $\bullet$ Drug treatment $\bullet$ Antioxidants $\bullet$ Insuin-sensitizers

- PPAR agonists • Anti-fibrotic agents

\section{KEY POINTS}

- There is a recognized clinical need for an effective treatment of nonalcoholic fatty liver disease (NAFLD); current approaches remain suboptimal and no drug has so far been approved by International Agencies.

- Several factors complicate the development of novel pharmacotherapies, particularly the imprecision of surrogate markers, making histologic assessment compulsory.

- Incretin mimetics, farnesoid x-receptor blockers, peroxisome proliferator activated receptor $\alpha / \delta$ agonists, and lysyl oxidase-like-2 inhibitory monoclonal antibodies are currently under scrutiny in randomized controlled trials.

- Although indicated by clinical guidelines, a careful follow-up and treatment of NAFLD is not the rule in the community. If, when, and how long drug therapy should be instituted and continued to reduce the burden of disease are being researched.

\section{INTRODUCTION}

Lifestyle changes are a mandatory strategy for the prevention and treatment of nonalcoholic fatty liver disease (NAFLD), but the results depend on individual subjects and therefore are largely unpredictable. Also, subjects who achieve a marked reduction

Funding Sources: Prof Marchesini: Funding from the European Community's Seventh Framework Programme (FP7/2007-2013) under grant agreement no. HEALTH-F2-2009-241762 for the project FLIP.

Conflict of Interests: Prof Marchesini: Advisory board Sanofi; Speaker's fee from Merck-Sharp and Dome, Lilly, NOVO Nordisk, Boehringer Ingelheim, Sanofi. Dr Mazzella, Dr Ricciardi, Dr Mazzotti: No conflict of interest.

${ }^{a}$ Unit of Metabolic Diseases \& Clinical Dietetics, Postgraduate School of Nutrition, S. Orsola-Malpighi Hospital, Alma Mater Studiorum University, Via Massarenti, 9, Bologna 40138, Italy; ${ }^{\text {b }}$ Unit of Metabolic Diseases \& Clinical Dietetics, S. Orsola-Malpighi Hospital, Via Massarenti, 9, Bologna 40138, Italy; ${ }^{C}$ Unit of Metabolic Disease \& Clinical Dietetics, S. Orsola-Malpighi Hospital, Alma Mater Studiorum University, Via Massarenti, 9, Bologna I-40138, Italy

* Corresponding author.

E-mail address: giulio.marchesini@unibo.it 
of body weight tend to regain weight along the years; in this case recurrence and/or progression of disease may be very likely. This finding stimulated intensive research on pharmacologic treatment strategies and several randomized controlled trials having histology as treatment outcome have been published. ${ }^{1-11}$ Several classes of drugs have been tested in the last 10 years, acting at different levels along the sequence of events from pure fatty liver to advanced disease (Fig. 1), but no drug has been so far been approved for the treatment of NAFLD. This finding opens a series of challenging questions that may be summarized, such as if, when, and how long should treatment be instituted/continued, considering that also with drugs the results are far from optimal? The situation is similar to that observed in other metabolic disorders largely linked to unhealthy lifestyles, namely, type 2 diabetes and obesity. International guidelines on the treatment of type 2 diabetes have never reached a general consensus as to the need to institute immediate pharmacologic treatment-with well-defined, effective, and safe drugs - soon after diagnosis, unless at risk of acute complications. In obesity all guidelines recommend systematic behavior treatment of weight loss before drug therapy-and very few drugs are approved by International Agencies. Drug therapy may also be effectively superimposed to drugs to increase the final results. ${ }^{12}$

The current scientific evidence on the principal drugs tested so far in several randomized controlled trials, divided according to their prevalent mechanism of action, is presented in Table 1 and is reviewed in this chapter.

\section{INSULIN SENSITIZERS}

As insulin resistance is the basis for liver fat accumulation, insulin sensitizers probably remain the best pharmacologic option for NAFLD treatment.

\section{Metformin}

Metformin is a biguanide used widely in clinical practice as a first-line treatment for patients with type 2 diabetes mellitus for over 50 years. Metformin reduces blood

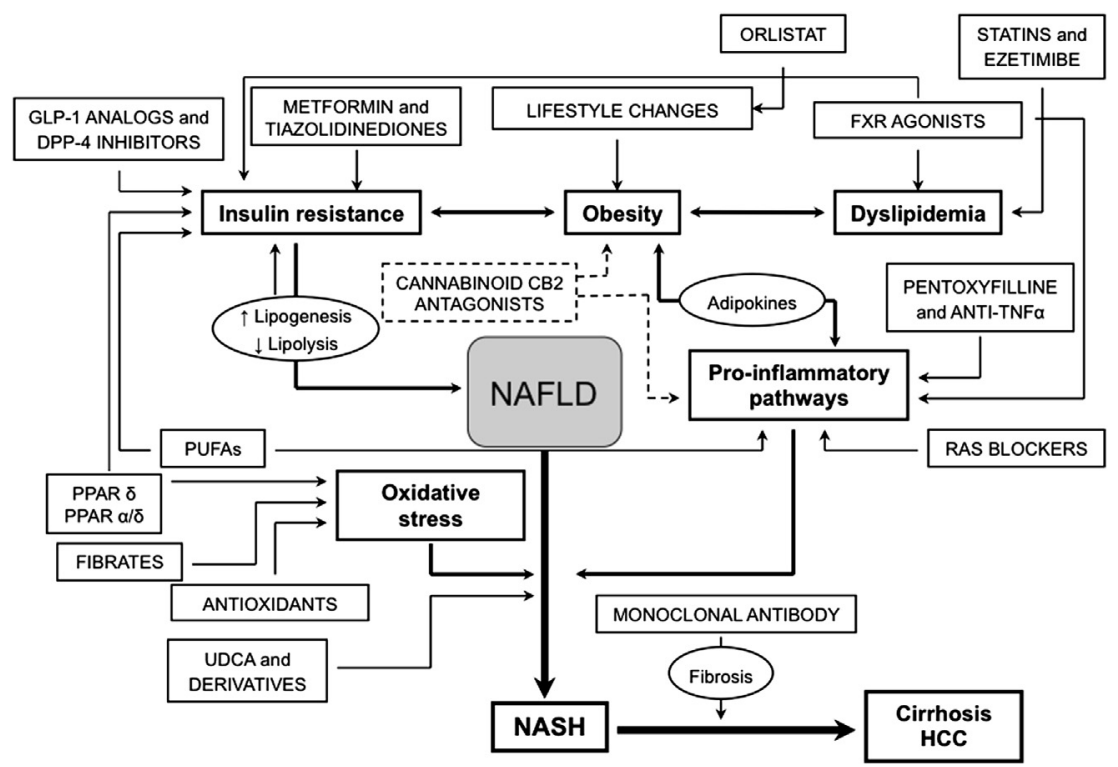

Fig. 1. The complex network of NAFLD pathogenesis and treatment. 


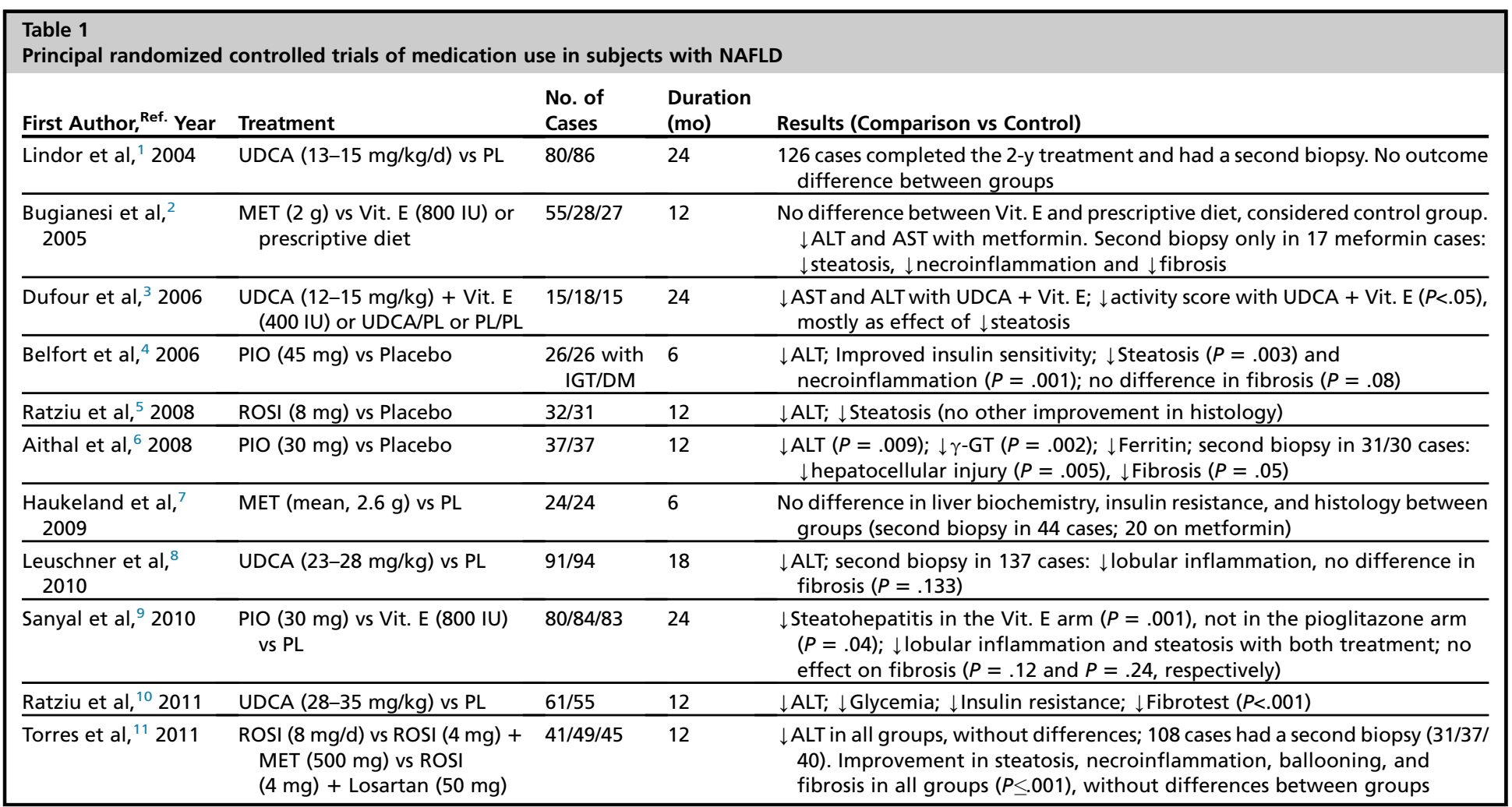

Abbreviations: DM, diabetes mellitus; IGT, impaired glucose tolerance; MET, metformin; PIO, pioglitazone; PL, placebo; ROSI, rosiglitazone.

Data from Refs. ${ }^{1-11}$ 
glucose by decreasing hepatic gluconeogenesis, by stimulating glucose uptake in the muscle, and by increasing fatty acid oxidation in adipose tissue. The final effect is an improvement of peripheral insulin sensitivity.

Following a seminal study in $2001,{ }^{13}$ a few clinical trials have reported a beneficial effect of metformin in NAFLD, but limited data are available on histology; metformin led to some improvements in steatosis and necroinflammation, but not in fibrosis. In most studies the changes seen with metformin were not different from those in the control arm and a recent systematic review concluded for a negative effect of metformin on histology. ${ }^{14}$ For this reason, the US Guidelines on NAFLD do not support metformin for the treatment of adult NAFLD. ${ }^{15}$

The potential role of metformin has also been examined in pediatric NAFLD patients with results similar to those observed in adults; metformin reduces liver enzymes and improves metabolic parameters, but not histologic features. ${ }^{16,17}$

Metformin treatment also promotes weight loss possibly via appetite control, which makes metformin the first-choice anti-diabetic medication for type 2 diabetes mellitus treatment in obese patients. However, it is unclear whether the benefits of metformin are greater than what might be achieved with weight loss from diet and exercise alone or with a weight loss medication that does not directly affect insulin sensitivity. ${ }^{18}$

The potential beneficial effects of metformin, however, extend outside liver fat. Metformin significantly decreases arterial stiffness, a marker of generalized atherosclerosis, associated with change in circulating adiponectin, a possible marker of the association between liver dysfunction and atherosclerotic vascular disease in patients with NAFLD. Furthermore, metformin has anticancer properties and is being tested to prevent primary cancer in several at-risk conditions. For all these reasons, metformin use might be re-evaluated in NAFLD.

\section{Glitazones (Thiazolidinediones)}

Thiazolidinediones (TZDs) have a significant effect on insulin sensitivity in insulinresistant states and in type 2 diabetes mellitus, as well as in patients with fatty liver or nonalcoholic steatohepatitis (NASH).

TZDs (troglitazone, rosiglitazone, and pioglitazone) are a class of peroxisome proliferator activated receptor $\gamma$ (PPAR- $\gamma$ ) agonists notable for the ability to cause differentiation of pluripotent stem cells into adipocytes. PPARs are predominantly expressed in adipose tissue, but are also present in muscle, liver, pancreas, heart, and spleen. TZDs treatment increases plasma adiponectin levels and has been shown in patients with type 2 diabetes and those with NASH. Patients with NASH have low plasma adiponectin levels, which are inversely related to insulin resistance and hepatic triglyceride content and are independent of the degree of obesity or glucose tolerance status; the increase in plasma adiponectin levels could mediate some of the insulinsensitizing effects of PPAR- $\gamma$ agonists, ${ }^{19}$ adding to their anti-inflammatory effects in patients with NASH.

TZDs are probably the best pharmacologic option for subjects with NAFLD. Three large randomized controlled trials reported a beneficial effect of pioglitazone on liver histology, although the advantage was limited for fibrosis. ${ }^{6,8,9}$ Rosiglitazone proved effective only on steatosis and liver enzymes, without an effect on necroinflammation and fibrosis. ${ }^{7}$ Continuing use of TZDs does not further improve the effects on histology, ${ }^{20}$ which are lost after treatment is stopped (Box 1). ${ }^{21}$

In conclusion, the efficacy of insulin sensitizers (particularly TZDs), strictly dependent on increased insulin sensitivity, is proven, although limited. Whether they need to be used in association with hepatoprotective agents in individual patients, to maximize the anti-inflammatory and antifibrotic activity, must be defined. There is now solid 
Box 1

Insulin sensitizers-mechanism of action

Metformin

- Activation of adenosine monophosphate-activated protein kinase, a regulator of energy metabolism

- Reduced hepatic gluconeogenesis via inhibition of the sterol regulatory element-binding protein-1c (SREBP-1c)

- Adipokine synthesis or secretion

Tiazolidinediones

- Adipocyte differentiation and adipogenesis

- Modification of adipose tissue distribution, with decreased visceral fat, including hepatic fat, and increased peripheral adiposity associated with weight gain

- "Browning" of adipose tissue mitochondria

- Stimulation of fatty acid oxidation and inhibition of hepatic fatty acid synthesis

- Improved insulin signaling and increase in adiponectin levels

evidence for their use, ${ }^{22}$ mitigated by undesired side effects (weight gain) and also adverse events.

\section{LIPID-LOWERING DRUGS, ANTIOXIDANT AND HEPATOPROTECTIVE AGENTS}

Several studies confirm a link between altered hepatocyte cholesterol metabolism and hepatic-free cholesterol accumulation and NAFLD development and progression. Dietary lipid intake is also an important cofactor in NAFLD development and progression, ${ }^{23}$ as in some genetic variants linked with lipid metabolism, like the patatin-like phospholipase domain-containing protein $3,{ }^{24}$ supporting the concept that drugs used for lipid control may be an effective treatment of NAFLD. ${ }^{25}$ Reducing lipid levels may also be important to reduce peroxidation, also achieved by different drugs.

The adipose tissue is considered a metabolically active endocrine organ producing pro-inflammatory cytokines, including tumor necrosis factor- $\alpha$ and interleukin- 6 and -8 , and there is evidence for the activation of other inflammatory pathways and oxidative stress, acting as a "second hit" in the transition between simple fatty liver and steatohepatitis (NASH). Excessive fat accumulation in the liver, whatever its cause, may increase the production of reactive oxygen species, leading to lipid peroxidation and immunologic dysfunction, which prompted testing the effectiveness of antioxidant and cytoprotective compounds, potentially stopping hepatocyte damage (Box 2).

\section{Statins}

By their activity on hydroxymethylglutaryl $\mathrm{CO}_{2}$ reductase, statins effectively reduce cholesterol levels in NAFLD in a dose-dependent manner, but their effects are not limited to cholesterol concentrations. Statins reduce the cardiovascular risk, the main cause of death in NAFLD, and control the inflammatory mechanisms involved in NAFLD pathogenesis. ${ }^{26,27}$

The use of statins in NAFLD received additional attention after the publication of the GREACE study, the first randomized controlled trial showing therapeutic benefit on clinical endpoints in NAFLD. ${ }^{23}$ In a post-hoc analysis, the use of statins in patients with high transaminase levels presumably due to NAFLD effectively reduced the 
Box 2

Lipid-lowering drugs, antioxidant and hepatoprotective agents-mechanism of action

- Decreased lipotoxicity and improved insulin sensitivity (lipid-lowering drugs)

- PPAR- $\alpha$ activity (fibrates)

- Reduced lipid peroxidation and free radicals scavenging activity (antioxidants)

- Anti-inflammatory properties, including the inhibition of pro-inflammatory cytokine production, translating into reduced apoptosis (pentoxyfilline)

- Modulation of inflammation and fibrogenesis and interference with intrahepatic glycolysis and gluconeogenesis (sylibin)

cardiovascular risk. Atorvastatin was the most widely used drug; pharmacokinetic differences translate into different effectiveness in preventing fibrosis of necroinflammation in NAFLD ${ }^{26}$ and also the absence of dyslipidemia. ${ }^{26}$

In NAFLD, statins improved liver enzyme levels, ${ }^{27,28}$ without any alleged risk of hepatotoxicity. ${ }^{27-29}$ Very few data are available on liver histology; in the only small randomized controlled trial with posttreatment histology, 1-year treatment with simvastatin had no significant effect. ${ }^{23}$ Pitavastatin did not improve the severity of hepatic steatosis, whereas atorvastatin improved the grade of steatosis, without conflicting results on fibrosis. ${ }^{24,27}$

\section{Ezetimibe}

Ezetimibe reduces the absorption of the cholesterol and its target is the Niemann-Pick C1-like 1 protein. This protein is located in the brush border of the intestine and in the liver and is a sterol transporter that is important for the absorption of the cholesterol in the enterocytes and hepatocytes. The excessive amounts of cholesterol are lipotoxic through activation of the liver $X$ receptor. Therefore, the inhibition of the Niemann-Pick C1-like 1 protein does not only lead to a reduced hepatic cholesterol accumulation, but also to decreased lipotoxicity.

Ezetimibe may be used without any restriction in patients with hepatic diseases. In subjects with NAFLD or NASH, ezetimibe reduced liver enzyme levels and the concentration of inflammatory markers ${ }^{27,30,31}$; in a few reports the histologic features of steatosis, ballooning, and the NAFLD activity score also improved. ${ }^{27,31,32}$ As to fibrosis, there is good evidence for improvement in animal models, but more data are needed in humans. ${ }^{31}$

\section{Fibrates}

Fibrates (fenofibrate, bezafibrate, gemfibrozil) effectively lower serum triglycerides and moderately increase high-density lipoproteins through binding to and activation of PPAR- $\alpha$.

PPAR- $\alpha$, member of the PPAR nuclear receptor subfamily, is highly expressed in the hepatocytes, where it controls genes involved in lipid and lipoprotein metabolism, including the uptake and oxidation of free fatty acids, triglyceride hydrolysis, and up-regulation of reverse cholesterol transport, mediated by apolipoprotein A-I and A-II. Furthermore, fibrates improve insulin sensitivity, stimulate fatty acid oxidation, and inhibit vascular inflammation.

Fenofibrate is commonly used in clinical practice to treat hypertriglyceridemia; in NAFLD it increases the expression of enzymes involved in the catabolism of lipid peroxides and reduces hepatic lipid peroxide content. ${ }^{33,34}$ Gemfibrozil decreases serum aminotransferase levels in patients with NAFLD, but no data are available on insulin 
resistance and liver histology. ${ }^{35}$ Bezafibrate, a PPAR pan-agonist, reduces hepatic lipids and the formation of proinflammatory lipoperoxides; along this line it might be particularly effective in NASH.

In conclusion, fibrates might be effective in NAFLD, at least in subjects with fasting hypertriglyceridemia, preventing lipid accumulation in the liver, $\mathrm{NASH}$, and fibrosis.

\section{Polyunsaturated Fatty Acids}

Polyunsaturated fatty acids (PUFA) are major constituents of cell membranes and are particularly susceptible to free radical-mediated oxidation. There is some evidence that a low intake of n-3 fatty acids may have a role in NAFLD pathogenesis, highlighting a potential therapeutic target.

When compared with controls, individuals with NAFLD have lower polyunsaturated fat intake. The composition of hepatic long chain fatty acids is characterized by a decrease in the relative levels of $n-3$ PUFA and an increase in the hepatic $n-6 / n-3$ PUFA ratio, ${ }^{36,37}$ associated with defective desaturation activity and dietary imbalance, resulting in hepatic steatosis. ${ }^{38,39}$

In humans, fish oil provides a convenient source of essential n-3 PUFA with few side effects and may directly reduce hepatic lipogenesis and steatosis, improving inflammation and hepatocyte injury. Given the well-recognized problems of adhering to lifestyle interventions and of achieving sustainable weight loss, and considering the side effects of pharmacologic agents, dietary fish oil supplementation represents a practical alternative therapy. ${ }^{37,40}$

In NAFLD, the dietary supplementation with long-chain n-3 PUFAs seems to reduce hepatic steatosis safely. ${ }^{41-43}$ A recent meta-analysis reported a statistically significant effect of PUFA supplementation on liver fat in 6/7 studies, a significant improvement of alanine aminotransferase (ALT) levels in 2, while aspartate aminotransferase (AST) was unaffected by PUFA. In 5 studies, steatosis was reduced by n-3 PUFA supplementation in the absence of weight loss. Fibrosis, hepatocyte ballooning, and lobular inflammation were reduced in $85 \%$ of the patients. ${ }^{43,44}$ Collectively, the data support a role for $n-3$ long-chain PUFA in NAFLD. The same results might be achieved by a diet rich in n-3 PUFA (fish, nuts, almonds, and other natural products).

\section{Orlistat}

Orlistat, a reversible inhibitor of gastric and pancreatic lipase, blocks the absorption of approximately $30 \%$ of dietary triglycerides. Orlistat improved AST/ALT, cholesterol, and triglyceride levels and reduced the grade of steatosis, inflammation, and fibrosis in an uncontrolled study. Two small trials in humans investigated the effect of orlistat in NAFLD, with negative results. ${ }^{45,46}$ Therefore, orlistat might be an effective treatment of $\mathrm{NASH}$ only in the setting of significant weight loss, possibly enhanced by a lifestyle program. $^{47}$

\section{Vitamin E}

Vitamin $\mathrm{E}$ is the most widely used antioxidant in biomedical research studies, but it is also linked to a greater risk of cardiovascular disease in epidemiologic studies.

Several studies have examined the role of supplemental vitamin $E$ in liver disease. Despite the encouraging in vitro work, results from clinical studies are conflicting. At doses of 400 to $1200 \mathrm{IU}$ daily, the administration of vitamin $\mathrm{E}$ reduces serum aminotransferases and alkaline phosphatase, both in monotherapy and as add-on to ursodeoxycholic acid (UDCA), and improves NASH, steatosis, and lobular inflammation, but not fibrosis scores, which are only improved by the association with vitamin C. ${ }^{9,48-50}$ The recent US Guidelines recommend vitamin $E$ and conclude that 
"Vitamin E administered at daily dose of 800 IU/day improves liver histology in nondiabetic adults with biopsy-proven NASH."15

\section{Pentoxifylline}

Pentoxifylline (PTX) is a methylxanthine derivative and a nonselective phosphodiesterase inhibitor with well-known hemorheologic activity and anti-inflammatory properties; it acts as a free radical scavenger, inhibits pro-inflammatory cytokine production, namely, tumor necrosis factor- $\alpha$ (TNF- $\alpha$ ), and reduces apoptosis.

In patients with NASH, PTX treatment for greater than 1 year versus placebo resulted in a statistically significant normalization or improvement of $30 \%$ or more in ALT but not in AST. ${ }^{51,52}$ In a systematic review including 6 trials, PTX treatment at a dose of $800 \mathrm{mg}$ to $1600 \mathrm{mg}$ per day for 3 to 6 months improved liver enzymes; histology was only improved after 12 months of follow-up. ${ }^{53}$ The positive effects on liver fibrosis might be the consequence of reduced oxidized lipid products. The overall methodological quality of the published studies is however relatively weak and larger studies are needed for additional validation. ${ }^{54}$

\section{Sylibin}

Silybin is a potent antioxidant representing about $50 \%$ to $70 \%$ of the silymarin extract of Silybum marianum (milk thistle). Silybin modulates inflammation and fibrogenesis and interferes with intrahepatic glycolysis and gluconeogenesis. As with other flavolignans, limitations of silybin use include low water solubility, low bioavailability, and poor intestinal absorption, but derivatives with improved solubility may overcome these pharmacologic limitations.

Silybin treatment attenuated liver damage and diabetes in animal models of NASH. The synthetic derivative in use in clinical practice is the silybin phytosome complex (silybin plus phosphatidylcholine), coformulated with vitamin $\mathrm{E}$, with much higher bioavailability.

In animal models silybin administration reduces insulin resistance and liver enzymes, as well as hepatic and myocardial damage, at doses similar to those used in humans. Considering the good tolerability of sylibin and its positive effects, further investigation is warranted.

\section{BILE ACIDS AND DERIVATIVES Ursodeoxycholic Acid (UDCA)}

The rationale for using UDCA (a tertiary bile acid) as a broad hepatoprotective agent is based on a large body of preclinical data ${ }^{55}$ and on controlled trials (Box 3). The
Box 3
Bile acids and derivatives-mechanism of action
- Hepatoprotective effect (UDCA)
- Anti-inflammatory action, mediated by decreased transcription of tumor necrosis factor- $\alpha$ (UDCA)
- Improved insulin sensitivity in muscle tissue and in the liver
- Down-regulation of lipogenic and apoptotic pathways (Nor-UDCA), favoring increased cholesterol efflux
- Protection against bile-salt-induced cellular toxicity (Tauro-UDCA)
- Anti-inflammatory and lipid-lowering activity (UDCA-LPE) 
histologic efficacy remains controversial but there is strong evidence of biochemical effectiveness (on ALT), arguing in favor of a broader hepatoprotective effect of UDCA.

Between 1994 and 2008, 4 studies on UDCA treatment were published on NASH. At doses of 12 to $15 \mathrm{mg} / \mathrm{kg} / \mathrm{d}$ UDCA monotherapy did not produce any significant effect on liver enzyme levels and histology ${ }^{1}$; the combination of UDCA and vitamin $E$ resulted in significant effects on histology. ${ }^{3}$ High-dose UDCA (28-35 mg/kg/d) versus placebo improved liver enzymes, glucose, and insulin levels, ${ }^{8,11}$ but the UDCA-treated group lost on average $3 \%$ of original body weight, possibly contributing to the favorable results.

Although UDCA monotherapy will not be further tested in NASH, UDCA derivatives have shown promising efficacy stronger than UDCA in preclinical models. In a genetic model of NASH, nor-ursodeoxycholic acid, a C23 homolog of UDCA, improved steatohepatitis by down-regulating lipogenic and apoptotic pathways while increasing hepatic cholesterol efflux. Tauro-ursodeoxycholic acid, a hydrophilic conjugate of UDCA, was able to block apoptosis, thus resulting in improved insulin resistance. Finally, a synthetic bile acid-phospholipid conjugate ursodeoxycholyllysophosphatidylethanolamide (UDCA-LPE) was designed to target phosphatidylcholine to hepatocytes by means of the bile acid transport systems. In in vivo models of $\mathrm{NASH}$, it reduced hepatic fat overload and inhibited de novo lipogenesis, also reducing proinflammatory pathways and liver enzyme levels.

$A$ recent study confirmed that UDCA-LPE ameliorates hepatic injury in different stages of NAFLD, such as steatosis and advanced steatohepatitis. For the excellent anti-inflammatory and lipid-lowering properties, and inhibition of disease progression, UDCA-LPE represents a promising compound suitable for the treatment of NAFLD. ${ }^{56}$

\section{NEW AREAS OF RESEARCH}

Several new areas of research are being exploited or old areas are receiving new interest and developments, to provide more effective and safer drugs for NAFLD treatment (Box 4).

\section{Box 4}

New areas of research-mechanism of action of new drugs

- Stimulation of the farnesoid X receptor- $\alpha$ that regulates glucose and lipid metabolism (OCA)

- Immunomodulatory and anti-inflammatory action, mediated by the inhibition of nuclear factor-kB and down-regulation of inducible nitric oxide synthase (OCA)

- Increased hepatic insulin signaling and sensitivity (GLP-1 agonists)

- Decreased hepatic lipogenesis and liver triglyceride content (GLP-1 agonists)

- GLP-1 agonist- and DPP-4 inhibitor-mediated protection of pancreatic $\beta$-cells from endoplasmic reticulum stress and apoptosis

- Insulin-sensitizing activity in the liver (PPAR- $\delta$ agonists)

- Reduced food intake (Endocannabinoid CB2 agonists)

- Improved insulin sensitivity and block of the hepatic recruitment of inflammatory cells and the development of fibrosis (ARB)

- Direct inhibition or even reversal of hepatic fibrosis (Lysyl oxidase-like-2 inhibitory monoclonal antibody) 


\section{Obeticholic Acid and Farnesoid X Receptor Agonists}

Bile acids are critical regulators of hepatic lipid and glucose metabolism through 2 major receptor pathways: farnesoid $X$ receptor (FXR), a member of the nuclear hormone receptor superfamily, and G protein-coupled bile acid receptor 1 (GPBAR1). FXRs are mainly found in the liver, kidney, and intestines, and overall inhibit hepatic bile acid production.

FXR knockout mice have high plasma triglyceride and cholesterol levels as well as a hepatic phenotype similar to NASH patients, ${ }^{57}$ including the possible development of hepatocellular carcinoma (HCC). ${ }^{58}$ Signaling through FXR and GPBAR1 modulates metabolic pathways, regulating not only bile acid synthesis and their enterohepatic recirculation but also triglyceride, cholesterol and glucose levels, energy homeostasis, and immune responses.

Obeticholic acid (OCA, INT-747, 6 $\alpha$-ethyl-chenodeoxycholic acid), a semisynthetic derivative of chenodeoxycholic acid, is a natural agonist of the FXR- $\alpha$ nuclear hormone receptor that regulates glucose and lipid metabolism. In animal models, OCA decreases insulin resistance and hepatic steatosis and displays immunomodulatory and anti-inflammatory properties. ${ }^{59}$ In a phase 2 trial, OCA administration for 6 weeks was well tolerated, increased insulin sensitivity, and reduced liver enzymes and the markers of liver inflammation and fibrosis in patients with type 2 diabetes and NAFLD. ${ }^{60}$ A large US multicenter, 18-month phase Ilb study of OCA in NASH patients is currently ongoing. Overall, adverse events were not different in patients on treatment or on placebo.

\section{Incretin Mimetics}

The rationale for the use of the glucagon-like peptide-1 analogues (GLP-1a) and the dipeptidyl peptidase-4 inhibitors (DPP-4i) in NAFLD does not only derive from their insulin-sensitizing activity but also from the evidence of a reduced activity of the incretin system in NASH patients. The expression of GLP-1 receptors in liver or hepatocytes is inconsistent in different laboratories, but the expression in the biopsies from NASH patients is generally lower compared with control biopsies, ${ }^{61}$ and DPP4 activity is $30 \%$ increased. ${ }^{62}$ Notably, both the serum activity and the intensity of DPP-4 immunostaining in the liver are associated with the intensity of fatty infiltration and histologic grading of NASH, providing a rationale for the use of DPP-4i to slow the progression of hepatic steatosis and inflammation. ${ }^{63} \mathrm{GLP}-1 \mathrm{a}$ and DPP-4i are also likely to improve NAFLD through improved insulin sensitivity. ${ }^{64}$

The protective effects of incretin-mimetic agents on hepatic steatosis were found in diet-induced obese mice treated with GLP-1 analogues and with DPP-IV inhibitors (in linagliptin-treated diet-induced obese mice liver fat content was reduced by up to $30 \%),{ }^{64-68}$ but data were not confirmed in patients treated with exenatide. ${ }^{69}$ More research is needed to explore the mechanism and the possibility of using incretinmimetic agents as therapy for NAFLD. ${ }^{70}$ Notably, clinical studies have provided evidence that DPP-4i can be used safely without any risk of hypoglycemia even in nondiabetic patients. ${ }^{63}$

\section{PPAR- $\delta$ Agonists}

The function of PPAR- $\delta$ has long been unrecognized. Now PPAR- $\delta$ seems to be the most promising of all PPAR targets for its specific action on the liver, muscle, and fat. The liver was only recently identified as a major PPAR- $\delta$-responsive tissue, able to burn large amounts of glucose, thus reducing hyperglycemia and improving insulin sensitivity. PPAR- $\delta$ also regulates the catabolism and/or the $\beta$-oxidation of lipids in 
adipose tissue and muscle, increases the production of mono-unsaturated fatty acids, and may protect the liver from free fatty acid-mediated lipotoxicity and inflammatory response. ${ }^{71,72}$

The lipogenic activity of PPAR- $\delta$ has also been observed in human studies. ${ }^{73}$ Ligands for PPAR- $\delta$ have been proposed to act as insulin sensitizers, based on improvements in standard glucose-tolerance tests. Studies based on long-term ligand treatment regimens show a significant weight loss and a decreased fat mass, conditions potentially responsible for increased insulin sensitivity. Along this line, the PPAR$\delta$ agonist GW0742 was reported to reduce hepatic steatosis and hyperglycemia. ${ }^{72}$ In mice fed the steatogenic metionine-choline-deficient diet, the PPAR- $\delta$ agonist GW501516 improved hepatic steatosis and reduced inflammation. ${ }^{74,75}$ Thus, PPAR$\delta$ might be helpful in $\mathrm{NASH},{ }^{76}$ but no selective PPAR- $\delta$ agonists are clinically available at present.

\section{PPAR- $\alpha / \delta$ Agonists}

GFT505 and its main active metabolites are PPAR modulators with preferential activity on human PPAR- $\alpha$ in vitro (half-maximal effective concentration) and with additional activity on human PPAR- $\delta$. After oral administration, it accumulates predominantly in the liver, with concomitant repression of pro-inflammatory and profibrotic genes. Preclinical and clinical data demonstrated that GFT505 treatment improves several metabolic parameters, including fasting plasma glucose and insulin sensitivity (homeostasis model of assessment-insulin resistance) in abdominally obese patients. ${ }^{77}$ This improvement in metabolic parameters supports its use in the treatment of hepatic steatosis and the results seem promising. GFT505 treatment decreased plasma concentrations of liver enzymes and had a protective effect on steatosis, inflammation, and fibrosis. ${ }^{78,79}$ A randomized, double-blind, placebo-controlled, 1-year phase Ilb study (ClinicalTrial.gov identifier NCT01694849) is currently ongoing and will assess the efficacy and safety of GFT505 in patients with histologically proven NASH. No serious adverse events have so far been reported.

\section{Endocannabinoids (Cannabinoid Receptor Blockers Type 1 and Type 2 (CB1 and CB2))}

The endocannabinoid system, involved in the regulation of food intake and body weight, represents a target for NASH therapy. ${ }^{80}$ Rimonabant was the first selective CB1 receptor blocker introduced into clinical practice. CB1 antagonism also improved obesity-associated dyslipidemia and insulin resistance to a greater extent than expected from weight loss. For this reason, different studies were planned in NAFLD, supported by studies in experimental animals. Unfortunately, the alarming incidence of central side effects, including severe depression, ${ }^{81}$ led to rimonabant withdrawal. Contrary to CB1, highly expressed in the brain, CB2 receptors are mainly expressed in the periphery, predominantly by immune cells, and play a key role in inflammatory processes possibly involved in the pathogenesis of obesity-associated insulin resistance and the progression of fatty liver to NASH. ${ }^{81}$ Modulation of CB2 receptors is thus emerging as a potential therapeutic strategy, and the development of peripherally acting CB1/CB2 antagonists remains an area of intense research. ${ }^{82}$

\section{Drugs Modulating the Renin-Angiotensin System (RAS)}

In the liver, chronic injury up-regulates the local tissue renin-angiotensin system, which contributes to the recruitment of inflammatory cells and the development of fibrosis. Angiotensin receptor blockers (ARBs) might reduce oxidative stress, attenuating the progression of hepatic fibrosis. In human studies, 2 ARBs (losartan and valsartan) reduced transaminase levels ${ }^{11,83}$; one reduced the grade of liver steatosis, 
fibrosis, and ballooning, but ARB use never did reach the clinical stage. Nonetheless, they are widely used, with a well-characterized safety profile, in the presence of comorbidities.

\section{Lysyl Oxidase-Like-2 Inhibitory Monoclonal Antibody}

Fibroblasts constitute the major cell type of the stromal compartment and contribute to tumor growth, angiogenesis, and fibrotic disease through paracrine signaling. The matrix enzyme lysyl oxidase-like-2 has an important role in the creation and maintenance of the pathologic microenvironment in cancer and fibrotic diseases. The inhibition of this enzyme by a lysyl oxidase-like-2 inhibitor monoclonal antibody (sintuzumab, GS-6624; Gilead Sciences, Foster City, CA, USA) is associated with reduced tumor volume in a mice model, probably due to a reduction of cross-linked collagenous matrix and activated fibroblasts. The use of this monoclonal antibody is also associated with the inhibition of transfer growth factor- $\beta$ signaling in fibroblasts and reduced porto-portal and porto-central fibrosis. This evidence is the basis for the development of a new class of drugs to be tested in several hepatic diseases characterized by advanced fibrosis/cirrhosis, to reduce directly the progression to fibrotic stage and/or to reverse stable fibrosis. ${ }^{84}$ At least 2 phase Ilb trials are at present recruiting participants for studies in advanced NASH with/without cirrhosis by the use of GS-6624, infused every 2 weeks for 96 weeks. Outcome results are expected by August 2015.

\section{SUMMARY}

There is a definite clinical need for an effective treatment of NAFLD, but current approaches remain suboptimal. Several factors will complicate the development of novel pharmacotherapies, including: (1) the multifactorial pathogenesis of NAFLD, (2) the heterogeneity of the patient population, (3) the imprecision of current disease staging techniques, (4) ill-validated surrogate markers, making histologic assessment compulsory, (5) the slowly progressive nature of NASH and the tendency of a proportion of cases to show spontaneous disease regression, likely related to the improvement of metabolic control. ${ }^{85}$

At present, no drugs have been approved with specific indications for NAFLD; there is however general consensus that continuing clinical research is needed on hard end points (ie, improvement or resolution of NASH), with no worsening of fibrosis and/or improvement of steatosis (quantitatively assessed) and sustained normalization of liver enzymes. ${ }^{86}$ Although indicated by clinical guidelines, a careful follow-up and treatment of NAFLD are not the rule in the community. Four questions remain unanswered: (1) Should drug therapy be initiated independently of lifestyle changes? (2) Which drug, if any, in individual patients, according to age, comorbidities, and disease severity? Which drug for NAFLD patients with diabetes, where most putative drugs are already in use, and in normal-weight NAFLD? (3) Should treatment be continued lifelong, in the absence of significant lifestyle changes?

Efforts should be made to close the gap and reduce the future burden of NAFLD and its complications. ${ }^{87}$

\section{REFERENCES}

1. Lindor KD, Kowdley KV, Heathcote EJ, et al. Ursodeoxycholic acid for treatment of nonalcoholic steatohepatitis: results of a randomized trial. Hepatology 2004; 39:770-8. 
2. Bugianesi E, Gentilcore E, Manini R, et al. A randomized controlled trial of metformin versus vitamin $\mathrm{E}$ or prescriptive diet in nonalcoholic fatty liver disease. Am J Gastroenterol 2005;100:1082-90.

3. Dufour JF, Oneta CM, Gonvers JJ, et al. Randomized placebo-controlled trial of ursodeoxycholic acid with vitamin E in nonalcoholic steatohepatitis. Clin Gastroenterol Hepatol 2006;4:1537-43.

4. Belfort R, Harrison SA, Brown K, et al. A placebo-controlled trial of pioglitazone in subjects with nonalcoholic steatohepatitis. N Engl J Med 2006;355: 2297-307.

5. Ratziu V, Giral P, Jacqueminet S, et al. Rosiglitazone for nonalcoholic steatohepatitis: one-year results of the randomized placebo-controlled Fatty Liver Improvement with Rosiglitazone Therapy (FLIRT) Trial. Gastroenterology 2008; 135:100-10.

6. Aithal GP, Thomas JA, Kaye PV, et al. Randomized, placebo-controlled trial of pioglitazone in nondiabetic subjects with nonalcoholic steatohepatitis. Gastroenterology 2008;135:1176-84.

7. Haukeland JW, Konopski Z, Eggesbo HB, et al. Metformin in patients with nonalcoholic fatty liver disease: a randomized, controlled trial. Scand J Gastroenterol 2009; 44:853-60.

8. Leuschner UF, Lindenthal B, Herrmann G, et al. High-dose ursodeoxycholic acid therapy for nonalcoholic steatohepatitis: a double-blind, randomized, placebocontrolled trial. Hepatology 2010;52:472-9.

9. Sanyal AJ, Chalasani N, Kowdley KV, et al. Pioglitazone, vitamin E, or placebo for nonalcoholic steatohepatitis. N Engl J Med 2010;362:1675-85.

10. Ratziu V, de Ledinghen V, Oberti F, et al. A randomized controlled trial of highdose ursodesoxycholic acid for nonalcoholic steatohepatitis. J Hepatol 2011;54: 1011-9.

11. Torres DM, Jones FJ, Shaw JC, et al. Rosiglitazone versus rosiglitazone and metformin versus rosiglitazone and Iosartan in the treatment of nonalcoholic steatohepatitis in humans: a 12-month randomized, prospective, open-label trial. Hepatology 2011;54:1631-9.

12. Wadden TA, Berkowitz RI, Womble LG, et al. Randomized trial of lifestyle modification and pharmacotherapy for obesity. N Engl J Med 2005;353:2111-20.

13. Marchesini G, Brizi M, Bianchi G, et al. Metformin in non-alcoholic steatohepatitis. Lancet 2001;358:893-4.

14. Vernon G, Baranova A, Younossi ZM. Systematic review: the epidemiology and natural history of non-alcoholic fatty liver disease and non-alcoholic steatohepatitis in adults. Aliment Pharmacol Ther 2011;34:274-85.

15. Chalasani N, Younossi Z, Lavine JE, et al. The diagnosis and management of non-alcoholic fatty liver disease: practice guideline by the American Gastroenterological Association, American Association for the Study of Liver Diseases, and American College of Gastroenterology. Gastroenterology 2012;142: 1592-609.

16. Mazza A, Fruci B, Garinis GA, et al. The role of metformin in the management of NAFLD. Exp Diabetes Res 2012;2012:716404.

17. Shyangdan D, Clar C, Ghouri N, et al. Insulin sensitisers in the treatment of nonalcoholic fatty liver disease: a systematic review. Health Technol Assess 2011; 15:1-110.

18. Loomba R, Lutchman G, Kleiner DE, et al. Clinical trial: pilot study of metformin for the treatment of non-alcoholic steatohepatitis. Aliment Pharmacol Ther 2009; 29:172-82. 
19. Gastaldelli A, Harrison S, Belfort-Aguiar R, et al. Pioglitazone in the treatment of NASH: the role of adiponectin. Aliment Pharmacol Ther 2010;32:769-75.

20. Ratziu V, Charlotte F, Bernhardt C, et al. Long-term efficacy of rosiglitazone in nonalcoholic steatohepatitis: results of the fatty liver improvement by rosiglitazone therapy (FLIRT 2) extension trial. Hepatology 2010;51:445-53.

21. Lutchman G, Modi A, Kleiner DE, et al. The effects of discontinuing pioglitazone in patients with nonalcoholic steatohepatitis. Hepatology 2007;46:424-9.

22. Ratziu V, Caldwell S, Neuschwander-Tetri BA. Therapeutic trials in nonalcoholic steatohepatitis: insulin sensitizers and related methodological issues. Hepatology 2010;52:2206-15.

23. Musso G, Cassader M, Gambino R. Cholesterol-lowering therapy for the treatment of nonalcoholic fatty liver disease: an update. Curr Opin Lipidol 2011;22: 489-96.

24. Schattenberg JM, Schuppan D. Nonalcoholic steatohepatitis: the therapeutic challenge of a global epidemic. Curr Opin Lipidol 2011;22:479-88.

25. Foster T, Budoff MJ, Saab S, et al. Atorvastatin and antioxidants for the treatment of nonalcoholic fatty liver disease: the St Francis Heart Study randomized clinical trial. Am J Gastroenterol 2011;106:71-7.

26. Dima A, Marinescu AG, Dima AC. Non-alcoholic fatty liver disease and the statins treatment. Rom J Intern Med 2012;50:19-25.

27. Nseir W, Mograbi J, Ghali M. Lipid-lowering agents in nonalcoholic fatty liver disease and steatohepatitis: human studies. Dig Dis Sci 2012;57:1773-81.

28. Tzefos M, Olin JL. 3-hydroxyl-3-methylglutaryl coenzyme A reductase inhibitor use in chronic liver disease: a therapeutic controversy. J Clin Lipidol 2011;5: 450-9.

29. Chatrath H, Vuppalanchi R, Chalasani N. Dyslipidemia in patients with nonalcoholic fatty liver disease. Semin Liver Dis 2012;32:22-9.

30. Chan DC, Watts GF, Gan SK, et al. Effect of ezetimibe on hepatic fat, inflammatory markers, and apolipoprotein B-100 kinetics in insulin-resistant obese subjects on a weight loss diet. Diabetes Care 2010;33:1134-9.

31. Filippatos TD, Elisaf MS. Role of ezetimibe in non-alcoholic fatty liver disease. World J Hepatol 2011;3:265-7.

32. Park H, Hasegawa G, Shima T, et al. The fatty acid composition of plasma cholesteryl esters and estimated desaturase activities in patients with nonalcoholic fatty liver disease and the effect of long-term ezetimibe therapy on these levels. Clin Chim Acta 2010;411:1735-40.

33. Fernandez-Miranda C, Perez-Carreras M, Colina F, et al. A pilot trial of fenofibrate for the treatment of non-alcoholic fatty liver disease. Dig Liver Dis 2008; 40:200-5.

34. Fabbrini E, Mohammed BS, Korenblat KM, et al. Effect of fenofibrate and niacin on intrahepatic triglyceride content, very low-density lipoprotein kinetics, and insulin action in obese subjects with nonalcoholic fatty liver disease. J Clin Endocrinol Metab 2010;95:2727-35.

35. Basaranoglu M, Acbay $O$, Sonsuz A. A controlled trial of gemfibrozil in the treatment of patients with nonalcoholic steatohepatitis. J Hepatol 1999;31:384.

36. Zelber-Sagi S, Nitzan-Kaluski D, Goldsmith R, et al. Long term nutritional intake and the risk for non-alcoholic fatty liver disease (NAFLD): a population based study. J Hepatol 2007;47:711-7.

37. Parker HM, Johnson NA, Burdon CA, et al. Omega-3 supplementation and nonalcoholic fatty liver disease: a systematic review and meta-analysis. J Hepatol 2013;56:944-51. 
38. Valenzuela R, Espinosa A, Gonzalez-Manan D, et al. N-3 long-chain polyunsaturated fatty acid supplementation significantly reduces liver oxidative stress in high fat induced steatosis. PLoS One 2012;7:e46400.

39. Araya J, Rodrigo R, Pettinelli P, et al. Decreased liver fatty acid delta-6 and delta-5 desaturase activity in obese patients. Obesity (Silver Spring) 2010;18:1460-3.

40. Zhu FS, Liu S, Chen XM, et al. Effects of n-3 polyunsaturated fatty acids from seal oils on nonalcoholic fatty liver disease associated with hyperlipidemia. World J Gastroenterol 2008;14:6395-400.

41. Shapiro H, Tehilla M, Attal-Singer J, et al. The therapeutic potential of long-chain omega-3 fatty acids in nonalcoholic fatty liver disease. Clin Nutr 2011;30:6-19.

42. Capanni M, Calella F, Biagini MR, et al. Prolonged n-3 polyunsaturated fatty acid supplementation ameliorates hepatic steatosis in patients with non-alcoholic fatty liver disease: a pilot study. Aliment Pharmacol Ther 2006;23:1143-51.

43. Spadaro L, Magliocco O, Spampinato D, et al. Effects of $n-3$ polyunsaturated fatty acids in subjects with nonalcoholic fatty liver disease. Dig Liver Dis 2008;40:194-9.

44. Tanaka N, Sano K, Horiuchi A, et al. Highly purified eicosapentaenoic acid treatment improves nonalcoholic steatohepatitis. J Clin Gastroenterol 2008;42: 413-8.

45. Zelber-Sagi S, Kessler A, Brazowsky E, et al. A double-blind randomized placebo-controlled trial of orlistat for the treatment of nonalcoholic fatty liver disease. Clin Gastroenterol Hepatol 2006;4:639-44.

46. Harrison SA, Fecht W, Brunt EM, et al. Orlistat for overweight subjects with nonalcoholic steatohepatitis: a randomized, prospective trial. Hepatology 2009;49:80-6.

47. Peng L, Wang J, Li F. Weight reduction for non-alcoholic fatty liver disease. Cochrane Database Syst Rev 2011;(6):CD003619.

48. Pietu F, Guillaud O, Walter T, et al. Ursodeoxycholic acid with vitamin E in patients with nonalcoholic steatohepatitis: Iong-term results. Clin Res Hepatol Gastroenterol 2012;36:146-55.

49. Lavine JE, Schwimmer JB, Molleston JP, et al. Treatment of nonalcoholic fatty liver disease in children: TONIC trial design. Contemp Clin Trials 2010;31:62-70.

50. Harrison SA, Torgerson S, Hayashi P, et al. Vitamin E and vitamin C treatment improves fibrosis in patients with nonalcoholic steatohepatitis. Am J Gastroenterol 2003;98:2485-90.

51. Zein CO, Yerian LM, Gogate P, et al. Pentoxifylline improves nonalcoholic steatohepatitis: a randomized placebo-controlled trial. Hepatology 2011;54:1610-9.

52. Satapathy SK, Sakhuja P, Malhotra V, et al. Beneficial effects of pentoxifylline on hepatic steatosis, fibrosis and necroinflammation in patients with non-alcoholic steatohepatitis. J Gastroenterol Hepatol 2007;22:634-8.

53. Lee YM, Sutedja DS, Wai CT, et al. A randomized controlled pilot study of Pentoxifylline in patients with non-alcoholic steatohepatitis (NASH). Hepatol Int 2008;2:196-201.

54. Zein CO, Lopez R, Fu X, et al. Pentoxifylline decreases oxidized lipid products in nonalcoholic steatohepatitis: new evidence on the potential therapeutic mechanism. Hepatology 2012;56:1291-9.

55. Ratziu V. Treatment of NASH with ursodeoxycholic acid: pro. Clin Res Hepatol Gastroenterol 2012;36(Suppl 1):S41-5.

56. Pathil A, Mueller J, Warth A, et al. Ursodeoxycholyl lysophosphatidylethanolamide improves steatosis and inflammation in murine models of nonalcoholic fatty liver disease. Hepatology 2012;55:1369-78. 
57. Kong B, Luyendyk JP, Tawfik O, et al. Farnesoid X receptor deficiency induces nonalcoholic steatohepatitis in low-density lipoprotein receptor-knockout mice fed a high-fat diet. J Pharmacol Exp Ther 2009;328:116-22.

58. Zhang Y, Edwards PA. FXR signaling in metabolic disease. FEBS Lett 2008;582: $10-8$.

59. Adorini L, Pruzanski M, Shapiro D. Farnesoid X receptor targeting to treat nonalcoholic steatohepatitis. Drug Discov Today 2012;17:988-97.

60. Mudaliar S, Henry RR, Sanyal AJ, et al. Efficacy and safety of the farnesoid $X$ receptor agonist obeticholic acid in patients with type 2 diabetes and nonalcoholic fatty liver disease. Gastroenterology 2013;145(3):574-82.e1.

61. Pedersen J, Holst JJ. Glucagon like-peptide 1 receptor and the liver. Liver Int 2011;31:1243-5.

62. Balaban YH, Korkusuz P, Simsek H, et al. Dipeptidyl peptidase IV (DDP IV) in NASH patients. Ann Hepatol 2007;6:242-50.

63. Yilmaz Y, Atug O, Yonal O, et al. Dipeptidyl peptidase IV inhibitors: therapeutic potential in nonalcoholic fatty liver disease. Med Sci Monit 2009;15:HY1-5.

64. Kern M, Kloting N, Niessen HG, et al. Linagliptin improves insulin sensitivity and hepatic steatosis in diet-induced obesity. PLoS One 2012;7:e38744.

65. Ding X, Saxena NK, Lin S, et al. Exendin-4, a glucagon-like protein-1 (GLP-1) receptor agonist, reverses hepatic steatosis in ob/ob mice. Hepatology 2006; 43:173-81.

66. Son JP, Son MK, Jun SW, et al. Effects of a new sustained-release microsphere formulation of exenatide, DA-3091, on obese and non-alcoholic fatty liver disease mice. Pharmazie 2013;68:58-62.

67. Mells JE, Fu PP, Sharma S, et al. Glp-1 analog, liraglutide, ameliorates hepatic steatosis and cardiac hypertrophy in C57BL/6J mice fed a Western diet. Am J Physiol Gastrointest Liver Physiol 2012;302:G225-35.

68. Shirakawa J, Fujii H, Ohnuma K, et al. Diet-induced adipose tissue inflammation and liver steatosis are prevented by DPP-4 inhibition in diabetic mice. Diabetes 2011;60:1246-57.

69. Kenny PR, Brady DE, Torres DM, et al. Exenatide in the treatment of diabetic patients with non-alcoholic steatohepatitis: a case series. Am J Gastroenterol 2010;105:2707-9.

70. Lee J, Hong SW, Rhee EJ, et al. GLP-1 receptor agonist and non-alcoholic fatty liver disease. Diabetes Metab J 2012;36:262-7.

71. Liu S, Hatano B, Zhao M, et al. Role of peroxisome proliferator-activated receptor $\{$ delta\}/\{beta\} in hepatic metabolic regulation. J Biol Chem 2011;286: $1237-47$.

72. Lee MY, Choi R, Kim HM, et al. Peroxisome proliferator-activated receptor delta agonist attenuates hepatic steatosis by anti-inflammatory mechanism. Exp Mol Med 2012;44:578-85.

73. Riserus U, Sprecher D, Johnson T, et al. Activation of peroxisome proliferatoractivated receptor (PPAR)delta promotes reversal of multiple metabolic abnormalities, reduces oxidative stress, and increases fatty acid oxidation in moderately obese men. Diabetes 2008;57:332-9.

74. Iwaisako K, Haimerl M, Paik YH, et al. Protection from liver fibrosis by a peroxisome proliferator-activated receptor delta agonist. Proc Natl Acad Sci U S A 2012; 109:E1369-76.

75. Nagasawa T, Inada Y, Nakano S, et al. Effects of bezafibrate, PPAR pan-agonist, and GW501516, PPARdelta agonist, on development of steatohepatitis in mice fed a methionine- and choline-deficient diet. Eur J Pharmacol 2006;536:182-91. 
76. Wu HT, Chen CT, Cheng KC, et al. Pharmacological activation of peroxisome proliferator-activated receptor delta improves insulin resistance and hepatic steatosis in high fat diet-induced diabetic mice. Horm Metab Res 2011;43: $631-5$.

77. Cariou B, Zair Y, Staels B, et al. Effects of the new dual PPAR alpha/delta agonist GFT505 on lipid and glucose homeostasis in abdominally obese patients with combined dyslipidemia or impaired glucose metabolism. Diabetes Care 2011; 34:2008-14.

78. Cariou B, Hanf R, Lambert-Porcheron S, et al. Dual peroxisome proliferatoractivated receptor alpha/delta agonist GFT505 improves hepatic and peripheral insulin sensitivity in abdominally obese subjects. Diabetes Care 2013;36: 2923-30.

79. Staels B, Rubenstrunk A, Noel B, et al. Hepato-protective effects of the dual PPARalpha/delta agonist GFT505 in rodent models of NAFLD/NASH. Hepatology 2013. [Epub ahead of print].

80. Kashi MR, Torres DM, Harrison SA. Current and emerging therapies in nonalcoholic fatty liver disease. Semin Liver Dis 2008;28:396-406.

81. Mallat A, Lotersztajn S. Endocannabinoids and their role in fatty liver disease. Dig Dis 2010;28:261-6.

82. Durazzo M, Belci P, Collo A, et al. Focus on therapeutic strategies of nonalcoholic fatty liver disease. Int J Hepatol 2012;2012:464706.

83. Yokohama S, Yoneda M, Haneda M, et al. Therapeutic efficacy of an angiotensin II receptor antagonist in patients with nonalcoholic steatohepatitis. Hepatology 2004; 40:1222-5.

84. Barry-Hamilton V, Spangler R, Marshall D, et al. Allosteric inhibition of lysyl oxidase-like-2 impedes the development of a pathologic microenvironment. Nat Med 2010;16:1009-17.

85. Schuppan D, Gorrell MD, Klein T, et al. The challenge of developing novel pharmacological therapies for non-alcoholic steatohepatitis. Liver Int 2010;30: 795-808.

86. Sanyal AJ, Brunt EM, Kleiner DE, et al. Endpoints and clinical trial design for nonalcoholic steatohepatitis. Hepatology 2011;54:344-53.

87. Nascimbeni F, Pais R, Bellentani S, et al. From NAFLD in clinical practice to answers from guidelines. J Hepatol 2013;59:859-71. 